\title{
4 Minimalinvasive schönheitschirurgische Eingriffe mit Botulinumtoxin und Fillern
}

\author{
Dagmar Scharschmidt
}

Die Nachfrage nach nicht chirurgischen oder minimalinvasiven Behandlungen gegen die sichtbaren Zeichen des Alterns steigt seit Jahren rapide an. Insbesondere die Anwendung von Botulinumtoxin zur Glättung mimischer Falten und sogenannter Dermalfiller, insbesondere Hyaluronsäure, zum Auffüllen von Falten und zur Volumengebung verzeichnen ein rasantes Wachstum. Die relativ unkomplizierte Anwendung und geringen Nebenwirkungen mit raschen und sehr beeindruckenden Ergebnissen machen die minimalinvasiven Verfahren zu sehr populären und weiter anwachsenden Behandlungsverfahren für eine breite Bevölkerungsgruppe.

\subsection{Geschichtliche Entwicklung bis hin zur ästhetischen Anwendung}

\subsubsection{Botulinumtoxin}

Botulinumtoxin ist das Exotoxin des anaeroben Bakteriums Clostridium botulinum, dessen Sporen ubiquitär verbreitet sind. Botulinumtoxin ist ein zweikettiges Polypeptid, bestehend aus einer leichten und einer schweren Kette. Es sind sieben serologische Formen, Typ A bis $\mathrm{G}$ bekannt. In der ästhetischen Medizin wird ausschließlich der Serotyp A verwendet. Botulinumtoxin wirkt an cholinergen Synapsen und hemmt die Sekretion von Acetylcholin an der neuromuskulären Endplatte aber auch an cholinergen vegetativen Synapsen. Dies führt zur Unmöglichkeit muskulärer Kontraktionen und somit zur Parese der entsprechenden Muskulatur, bzw. an vegetativen Synapsen zur Reduktion der Schweiß- oder Speichelbildung. 
Der württembergische Arzt Justinus Kerner (1786-1862, Arzt und Schriftsteller) sammelte um 1800 herum die Krankengeschichten von 155 Patienten mit Botulismus, einer durch verdorbene Wurstwaren hervorgerufenen Lebensmittelvergiftung, die zu schweren Erkrankungen und Todesfällen geführt hatte. Anhand von beschlagnahmten Wurstwaren erforschte er die Wirkung des Giftes an Kleintieren und schlug schon damals eine mögliche medizinische Behandlung von muskulären Überbewegungen mittels des Giftes vor.

1897 wurde das Gift durch den belgischen Arzt Prof. van Ermengen erstmals charakterisiert. Ihm gelang es, aus verdorbenem Schinken einen anaeroben sporenbildenden Bazillus zu züchten. Daher ergibt sich auch der Name von Botulinumtoxin vom lateinischen botulus (Wurst) und toxin (Gift).

1897 wurde das erste Antiserum gewonnen, 1910 wurde der zweite Serotyp (B) entdeckt. Ab den 192oern wurde an der Universität California in San Francisco und später während des 2. Weltkrieges in Fort Detrick, Maryland, weiter über Herstellung und Wirkung des Toxins geforscht. 1949 wurde erstmals die Blockade der neuromuskulären Transmission als Wirkmechanismus des Giftes beschrieben. Nach mehreren tiermedizinischen Versuchen wurde synthetisiertes Botulinumtoxin 1978 erstmals an freiwilligen Probanden eingesetzt.

1980 veröffentlichte Allan B. Scott, Forscher an der Smith-Kettlewell Eye Research Foundation, seine Arbeit über den ersten therapeutischen Einsatz von Botulinumtoxin A zur Behandlung des Strabismus. Es folgte der Einsatz bei diversen Dystonien (z.B. Toticollis-, Beinspastiken) und Nystagmus. 1989 erfolgte durch die FDA die Zulassung zur Therapie des Strabismus, des hemifazialen Spasmus und Blepharospasmus.

Jean Carruthers, Professorin für Ophtalmologie an der Universität Vancouver in Kanada hatte seit 1982 an den klinischen Versuchen mit Botulinumtoxin über Strabismus teilgenommen. Von einer ihrer Patientinnen wurde sie auf die gleichzeitige Besserung ihrer Gesichtsfalten hingewiesen. Gemeinsam mit ihrem Ehemann Alastair Carruthers, Professor für Dermatologie an der Universität Vancouver, folgten nun systematische Behandlungen der Glabellaregion und später weiterer Regionen mimischer Falten mittels Botulinumtoxin. 1990 erschien die erste Publikation über die Anwendung von Botulinumtoxin in der kosmetischen Dermatologie (Carruthers u. Carruthers 1990). In den folgenden Jahren führten das Ehepaar Carruther und andere ärztliche Kollegen in den USA und England weitere Studien zur klinischen Wirksamkeit in anderen Gesichtsregionen sowie Dosiswirkungsstudien der beiden zum damaligen Zeitpunkt kommerziell erhältlichen Präparate Dysport ${ }^{\circledR}$ und Botox ${ }^{\circledR}$ durch .

2002 erfolgte die Zulassung für die Behandlung der Glabellafalte durch die FDA. Der rasche klinische Erfolg, die relativ einfache Anwendung und die geringen Nebenwirkungen führten in den Folgejahren zu einer weltweiten rasanten Verbreitung der ästhetischen Anwendung von Botulinumtoxin zur Behandlung mimischer Gesichtsfalten. 


\subsubsection{Hyaluronsäure (HS)}

Hyaluronsäure ist eine körpereigene Substanz und findet sich in chemisch identischer Form in den Geweben und Körperflüssigkeiten aller Wirbeltiere, also auch des Menschen. Der größte Teil findet sich in der Haut, in Dermis und Epidermis. Es erfüllt mannigfaltige biologische Aufgaben, so u.a. im Bindegewebe und bei der Zellmigration und -proliferation. Hyaluronsäure ist durch seine hohe Wasserbindungsfähigkeit ein wichtiger Bestandteil des Glaskörpers und der Tränenflüssigkeit des Auges, sowie der Gelenkflüssigkeit.

Hyaluronsäure gehört chemisch zu den Glykosaminglykanen, einer Untergruppe der Polysaccharide. Eine wichtige Eigenschaft, die in der ästhetischen Medizin genutzt wird, ist ihre Viskoelastizität.

In der Augenheilkunde wurde HS in unvernetzter Form schon seit den 6oerJahren eingesetzt. 1964 gelang die Herstellung von HS-Gel in vernetzter Form. Erst 25 Jahre später erfolgte die Anwendung von vernetzter HS im Bereich der Gelenke.

In der ästhetischen Medizin wird HS in hochgereinigter, nativer Form zur Stimulation der Bindegewebszellen, insbesondere der Fibroblasten genutzt. Klinische Studien zeigten eine Zunahme der Elastizität und des Hautturgors, sowie der Hautdicke. In chemisch vernetzter Form und bei höherer Konzentration liegt die HS als unlösliches Gel vor und wird in der ästhetischen Medizin als Volumengeber oder sogenannter dermal filler eingesetzt. Der erste Hyaluronsäurefiller zur Faltenauffüllung kam 1996 auf den Markt.

Wurden die ersten Hyaluronsäuren noch aus Hahnenkamm gewonnen, werden sie heute von Bakterien synthetisiert und besitzen kein allergenes Potenzial. Eine Vorabtestung ist nicht notwendig. Der Abbau erfolgt über körpereigene Hyaluronidasen und der Volumeneffekt besteht zwischen 6-12 Monaten.

Gesichtsfalten mit Fillern bzw. Füllmaterialien zu behandeln versuchte man schon seit vielen Jahrzehnten. Seit Anfang der 2oer-Jahre kamen Materialien wie menschliches Fett, später aber auch Paraffinöl und Silikon zum Einsatz. Wegen teilweise gravierender Nebenwirkungen wurden letztere jedoch relativ schnell wieder verboten (Wiest 2007).

1977 wurde erstmals ein Filler aus Rinderkollagen von der FDA zugelassen. Wegen dessen immunogenen Potenzials war eine Vortestung notwendig. Als erster Hyaluronsäure-Filler erhielt 1996 Restylane ${ }^{\circledR}$ auf dem europäischen Markt eine Zulassung.

Injizierbare Füllmaterialien wie Hyaluronsäure gehören nicht zu den verordnungspflichtigen Arzneimitteln sondern sind Medizinprodukte. Sie dürfen in der EU nur produziert und vertrieben werden wenn sie eine CE-Kennzeichnung besitzen, wobei verschiedene Anforderungen dafür erfüllt sein müssen. Medizinprodukte werden je nach potenziellem Risiko für den menschlichen Kör- 
per in unterschiedliche Klassen eingeteilt. Injizierbare Füllmaterialien gehören der Klasse IIb/III an und müssen nicht notwendigerweise einer klinischen Prüfung unterzogen werden um eine CE-Zertifizierung zu erhalten. So ist es ausreichend, wenn der Hersteller die Sicherheits- und Qualitätsnachweise anhand von Literaturanalysen erbringt.

Auf dem deutschen Markt gibt es derzeit ca. 120 verschiedene Filler mit CEZulassung.

Die stetig zunehmenden Absatzzahlen für ästhetische Behandlungen mit Fillern führen zu einem stark umkämpften Markt, wo neue HS-Filler mit großen Versprechungen und geringen Einkaufskosten beworben werden. Firmenfusionen und -aufkäufe, neue Namen für alte Produkte führen zu Verwirrungen im Dschungel der Präparate.

Validierte klinische Studien, Qualität der Herstellung und Konzentration der Inhaltsstoffe verursachen aber Kosten.

Der behandelnde Arzt sollte genau nachfragen, ob klinische Studien für sein Produkt vorliegen. Seriöse Firmen bieten ihren Anwendern „hands on workshops" an, wo neben dem theoretischen Wissen die spezifischen und produkteigenen Injektionstechniken von erfahrenen Ärzten erlernt werden können.

Einige nichtresorbierbare permanente, also nicht abbaubare Fillermaterialien aus Partikeln, u.a. Acrylamide, Acrylate oder deren Gemische mit Hyaluronsäure kamen Ende des letzten, Anfang diesen Jahrtausends mit teils großen Versprechen auf den Markt. Teilweise gravierende Nebenwirkungen und Komplikationen, die häufig noch nach vielen Jahren auftreten können, wie z.B. Fremdkörperreaktionen mit knotigen Verhärtungen, Fistelbildungen haben dazu geführt, dass sämtliche ästhetisch-chirurgischen Fachgesellschaften sich gegen deren Verwendung aussprachen.

\subsection{Häufigkeiten der Anwendung von Botulinum und Hyaluronsäure}

Immer mehr Menschen lassen minimalinvasive ästhetische Eingriffe an sich vornehmen. In den Medien sehen wir immer mehr scheinbar alterslose bzw. sehr jugendlich alternde Menschen. Ein häufiger Gedanke beim Fernsehen oder in Illustrierten blättern: Vor 20 Jahren sah die aber schon genauso aus. Und der Schein trügt oft nicht.

Genaue Daten, wie viele Menschen in einem Land minimalinvasive Eingriffe vornehmen lassen, liegen nicht vor. Einzelne Fachgesellschaften befragen jedoch ihre Mitglieder dazu. In Deutschland tut dies die Gesellschaft für Ästhetische Chirurgie Deutschland e.V., kurz GÄCD. Für den amerikanischen Markt liegen jährliche Statistiken durch die ASAPS, der American Society for Aesthetic Plastic Surgery, vor. Die Daten der GÄCD stammen von den ca. 430 Mitgliedern der Gesellschaft (GÄCD 2011). Für 2010 ergab die Auswertung 
70.460 Botulinumanwendungen bei Frauen und 9.237 bei Männern. Filler wurden 49.328-mal bei Frauen und 4.627-mal bei Männern injiziert. Im Jahre 2009 waren es 62.795 Botulinuminjektionen und 67.897 Behandlungen mit Fillern. Bei den Männern erfolgten 8.380 Behandlungen mit Botulinum und 5.039 mit Fillern. Laut GÄCD haben im Jahre 2009 die Behandlungen mit Fillern und Hyaluronsäure um 50\% im Vergleich zum Vorjahr zugenommen. Der Anstieg im Jahre 2010 scheint sich im Vergleich zum Vorjahr zu verringern.

Die Statistiken der ASAPS zeigen ebenfalls zunehmende Behandlungszahlen (ASAPS 2009, 2010, 2011). Allerdings hatten hier die stärksten Zunahmen schon vor einigen Jahren stattgefunden. So wurden im Jahr 199765.157 Botulinumbehandlungen durchgeführt, im Jahre 2002 waren es 1.658.667 Behandlungen und im Jahre 2011 2.619.739. 2011 zeigte damit eine Zunahme von 7,5\% gegenüber 2010 (2.437.165 Behandlungen) und eine 3.920,7\% Zunahme gegenüber 1997. Noch im Jahre 2005 waren es allerdings 3.294.782 Botulinumbehandlungen gewesen. Danach zeigte sich eine stetige Abnahme an Botulinumbehandlungen, von 3.181.592 Botulinumbehandlungen im Jahre 2006 auf 2.464 .123 Behandlungen im Jahre 2008 (s. Abb. 1). Eine ähnliche Entwicklung zeigten auch die Zahlen zur Anwendung von Hyaluronsäurefillern.

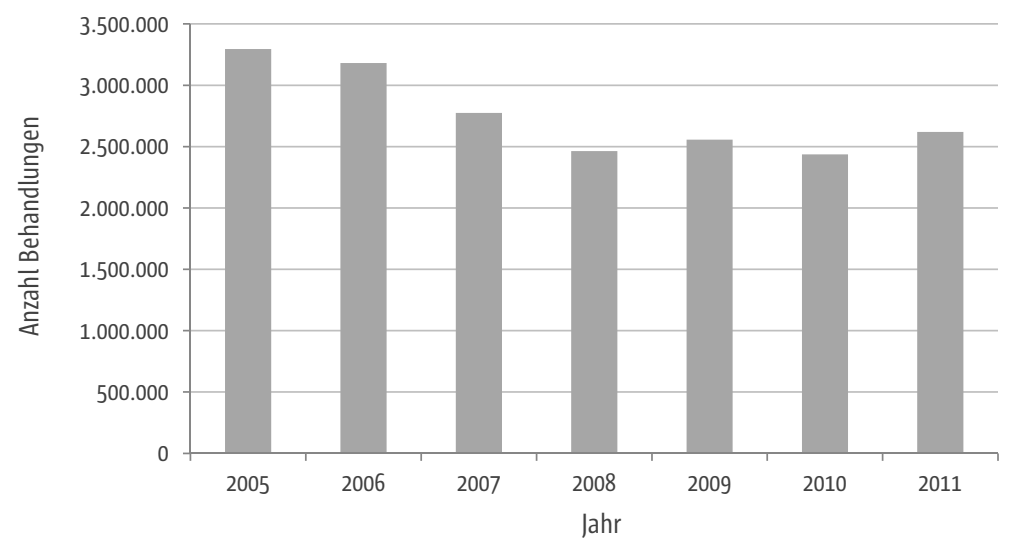

Abb. 1 Entwicklung der Behandlungszahlen ästhetischer Botulinumtoxin-Anwendungen (Daten der ASAPS)

Die Gründe für die zwischenzeitliche Abnahme der Behandlungszahlen für minimalinvasive ästhetische Maßnahmen dürften wirtschaftlicher Natur sein, da diese zeitgleich mit der Banken- und Immobilienkrise stattfanden. Andere Ursachen für die rückläufigen Behandlungszahlen, wie medizinische oder persönliche Gründe (zurück zum „natural look“) dürften auch aufgrund der wieder steigenden Anwendungen vernachlässigbar sein.

In Deutschland zeigen sich nach GÄCD-Daten weitere, wenn auch geringe Zunahmen für Behandlungen mittels Botulinum und Fillern. Bemerkt werden 
soll allerdings, dass immer mehr Ärzte der verschiedensten Fachrichtungen Behandlungen mit Botulinum und Fillern vornehmen. Weiterhin werden diese Behandlungen auch von Heilpraktikern und teilweise von Kosmetikerinnen durchgeführt.

Genaue Vergleichsdaten für die letzten 10 Jahre oder von größeren Anwendergruppen liegen für Deutschland nicht vor.

\subsection{Motive der Anwender bzw. Patienten}

Validierte klinische Untersuchungen oder Studien zur Motivation einer Behandlung mit Botulinum oder Fillern liegen kaum vor.

Einzig eine Untersuchung aus dem Jahre 2005 im Auftrag der Firma Allergan, die die Botulinumpräparate Botox ${ }^{\circledR}$ und Vistabe ${ }^{\circledR}$ herstellt, und der Aesthetic Surgery Education and Research Foundation (ASERF), fand sich dazu. Diese führten eine Befragung zur Motivation und der demografischen Verteilung der mit Botulinum Behandelten durch (ASERF 2005). Hierzu wurde ein zweiseitiger Fragebogen an 1.665 ASAPS-Mitglieder verschickt wobei 1.048 Fragebögen von Patienten ausgewertet werden konnten.

Dabei gaben 30\% der Befragten an, entspannter und weniger gestresst aussehen zu wollen, $24 \%$ wollten jünger aussehen. $17 \%$ führten die Behandlung durch, um weniger zornig bzw. streng auszusehen. $12 \%$ wollten mehr so aussehen, wie sie sich fühlten. Nur 13\% gaben an, attraktiver aussehen zu wollen (s. Abb. 2). Nach der Behandlung fühlten sich $47 \%$ attraktiver, $33 \%$ selbstbewusster und 29\% weniger gestresst. Die Untersuchung ergab weiterhin, dass die typische Anwenderin eine verheiratete Frau zwischen 40 und 55 Jahren ist. 97\% der Anwenderinnen waren Frauen, 81\% davon berufstätig. 44\% hatten ein Haushaltseinkommen unter 100.ooo Dollar. Vergleichende europäische oder deutsche Studien dazu liegen zurzeit noch nicht vor.

Wird in den deutschen Studien zur Wirksamkeit der Botulinum- oder Filleranwendung auch die Frage zur Zufriedenheit mit dem ästhetischen Ergebnis gestellt, gaben jedoch mehr als 80\% an, mit dem Ergebnis zufrieden bis sehr zufrieden zu sein und 100\% würden die Behandlung weiterempfehlen (Sommer et al. 2003).

In einer Untersuchung von Lewis fühlten sich die mit Botulinum behandelten Patienten bei der Befragung mittels IDAS (Irritability-Depression-Anxiety-Skala) signifikant glücklicher (Lewis u. Bowler 2009).

Es ist anzunehmen, dass sich deutsche oder europäische Anwender wenig in ihrer Motivation für minimalinvasive ästhetische Behandlungen von amerikanischen Anwendern unterscheiden.

In unserer Medien- und Dienstleistungsgesellschaft wird häufig eine zunehmende öffentliche Präsenz verlangt, und damit erlangt auch das eigene kör- 
perliche Erscheinungsbild eine immer stärkere Wertigkeit. Beispielsweise müssen Manager heute die Vitalität ihres Unternehmens und die eigenen Fähigkeiten auch durch ihr eigenes Aussehen widerspiegeln, wobei Behandlungen mit Botulinum und Fillern als hilfreich betrachtet werden.

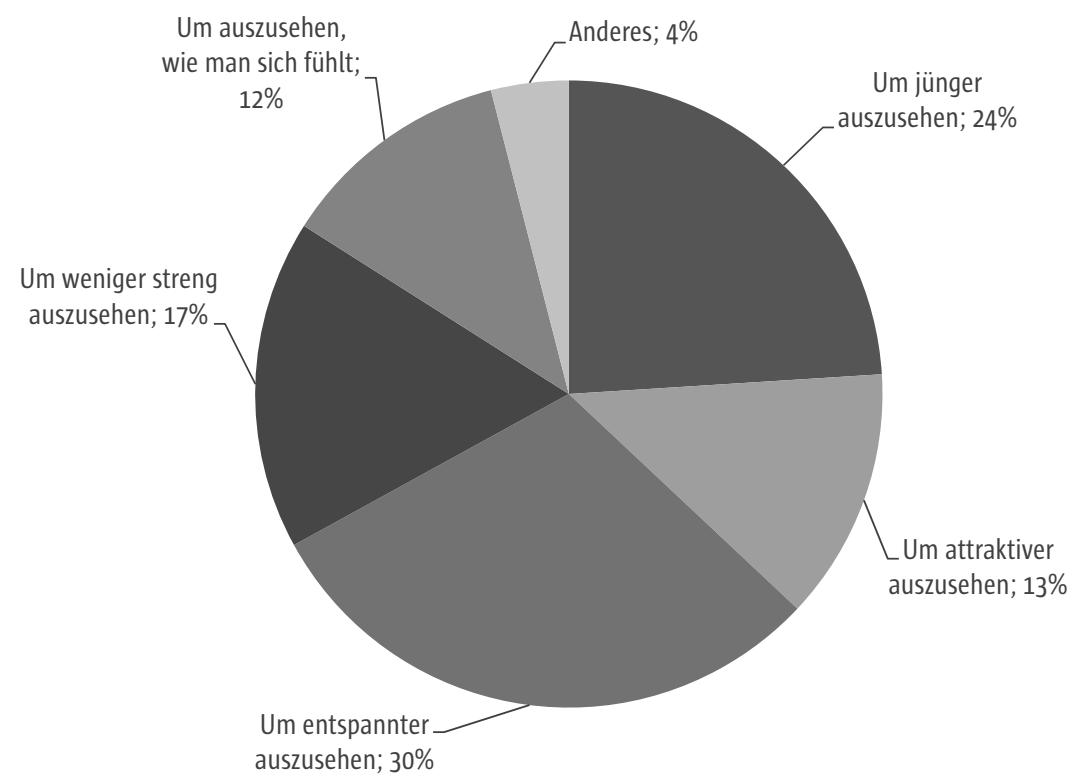

Abb. 2 Angaben zur Motivation für eine Botulinumbehandlung (Botox Cosmetic Survey 2005)

\subsection{Ausprägungen und gängige Formen}

Schönheit liegt im Auge des Betrachters, oder eben in dem des Patienten oder/ und des Arztes. Daher gibt es bei dieser Art der Körpermodifikation keine klassische Ausprägung oder gängigen Formen.

Natürlich gibt es Übertreibungen oder über der gängigen Norm liegende Ausprägungen, aber das sind, auch mit Hinweis auf die millionenfache Anwendung von Botulinum und Fillern doch eher die Ausnahmen und wesentliche Veränderungen des Aussehens sind mit diesen beiden ästhetischen Maßnahmen auch nicht möglich.

Wurde Botulinum in den ersten Jahren der ästhetischen Anwendung noch sehr schematisch und starr gespritzt, wird die Botulinumbehandlung, unter Einhaltung anatomischer Gegebenheiten, immer individualisierter angewendet.

Wie stark bzw. wie vollständig die Mimik des Gesichtes durch Botulinum ausgeschaltet wird, wobei eine vollständige Lähmung nur im oberen Gesichtsdrittel möglich ist, entscheiden letztendlich der Patient und der behandelnde 
Arzt. Wird z.B. nur die Glabella, also die Zornesfalte behandelt, ergibt sich lediglich ein entspannter Gesichtsausdruck ohne dass der Patient wesentlich auffällig oder anders aussieht. Ist die Mimik des gesamten oberen Gesichtes, also auch der Stirn- und lateralen Lidfalten gelähmt, ist dies meist nur erkennbar, wenn es nicht mit der übrigen Mimik oder dem dem Alter entsprechenden Bild des übrigen Gesichtes zusammenpasst oder zu starr gespritzt wurde.

Der untere Gesichtsbereich, insbesondere die Mundregion mit ihren mimischen Falten kann mit Botulinum nicht vollständig ruhiggestellt werden, da die dortigen Muskelgruppen Funktionen wie Sprechen oder Essen erfüllen müssen. Eine Anwendung von Botulinum zur Reduzierung mimischer Aktivität ist aber auch hier möglich.

Das Hauptanwendungsgebiet der HS ist die Auffüllung der statischen Falten. Tiefe eingegrabene Nasolabialfalten oder die radiären Lippenfalten sind klassische Indikationsbereiche. Natürlich ist auch hier eine übertriebene und auffällige oder zu häufige Anwendung möglich.

In den letzten Jahren hat sich der Blick der ästhetischen Anwender auf das zu behandelnde Gesicht geändert. Weg von der eindimensionalen Falte oder der eindimensionalen Betrachtung einer Falte zu einem dreidimensionalen Bild, dem „3D oder full face approach“. Berücksichtigt werden beim „,full face approach “ erstens Falten und Linien, zweitens Verlust von Volumen und Kontur, drittens Hautstruktur und Erscheinungsbild. Die ganzheitliche Herangehensweise kombiniert verschiedene Verfahren wie Botulinum und Filler, aber auch Laserbehandlungen und führt zu einem natürlicheren und harmonischeren Erscheinungsbild. Auch Hals, Dekolleté und Handrücken können dabei in die Behandlung mit eingeschlossen werden.

Beim „full face approach“ werden die individuellen Problemregionen des Patienten berücksichtigt und die Behandlung speziell auf die unterschiedlichen Falten bzw. Alterserscheinungen ausgerichtet. So können heute mit Fillern der neuen Generation auch stärkere Volumendefizite der oberen Wangenregion wieder angehoben werden oder abgesunkene Tränenrinnen wieder aufgefüllt werden. Ein Therapieplan mit der Anwendung von Botulinum für die Zornesfalte, dem Aufpolstern der Nasolabialfalte und einem Ausgleich des Volumenverlustes im Wangenbereich kann zu einem entspannten und frischen, dabei jedoch sehr natürlichen Ergebnis führen.

Den meisten Patienten wird man die Behandlung mit Botulinum und Fillern also nicht unbedingt ansehen und besonders in Deutschland legen die Patienten auch Wert auf ein natürliches Ergebnis.

Weiterhin ist anzumerken, dass Botulinum und Hyaluronsäurefiller wieder abgebaut werden und ihre Wirkung mit der Zeit nachlässt. Botulinum hat eine Wirkung von 4-6 Monaten, Hyaluronsäure von 6-12 Monaten im Mittel. Ob danach erneut behandelt werden soll, ist jedem selbst überlassen. 


\subsection{Medizinische Aspekte und Nebenwirkungen}

Botulinumtoxin ist bei fachgerechter Anwendung ein Medikament mit einem hohen Sicherheitsprofil. In der ästhetischen Behandlung werden dabei vielfach geringere Dosen eingesetzt als bei medizinischen Indikationen.

In der ästhetischen Behandlung liegen für alle drei in Deutschland verwendeten Produkte (Vistabel ${ }^{\circledR}$ Fa. Allergan, Azzalure ${ }^{\circledR}$ Fa. Galderma, Bocouture ${ }^{\circledR}$ Fa. Merz) nur Zulassungen für die Behandlung der Clabellaregion vor. Die Anwendung im übrigen Gesicht erfolgt im „off label use“ und der Patient ist darüber zu informieren. Für alle ästhetischen Botulinumprodukte liegen neben den Zulassungsstudien weitere Studien zur Sicherheit und Dosis vor.

Außerhalb der Anwendung in der Ästhetik nehmen die medizinischen Indikationen für Botulinumtoxin zu. Neue Indikationsfelder in der Neurologie, Urologie und weiteren Fachbereichen sind hinzugekommen. Neben der Anwendung bei vielen Spastiken (u.a. Hemifazialer Spasmus, Tics), Dystonien (zervikale Dystonie, Blepharospasmus u.a.) und Tremor wird es auch in der Urologie (neurogene Blasenentleerungsstörung, Dranginkontinenz) und bei vegetativen Störungen (Hyperhidrose, Hypersalivation u.a.) eingesetzt. Zunehmend wird es auch bei verschiedenen Schmerzsymptomen (z.B. Fibromyalgie) angewendet. Für die Behandlung der Migräne erfolgte im September 2011 die Zulassung in Deutschland. Dabei erfolgt die Behandlung mit Botulinum häufig auch für die medizinischen Indikationen im „off label use“. Eine Studie vom Februar 2012 zeigte eine Verbesserung von Depressionen nach der Behandlung der Clabellaregion mit Botulinum bei Patienten mit medikamentös ungenügend therapierbarer Depression (Wollmer et al. 2012).

Die Nebenwirkungen der ästhetischen Behandlung mit Botulinum und HSFillern sind gering. Kurzfristig gehören Schwellungen, Rötungen und Hämatome zu den häufigsten Nebenwirkungen. Seltenere Nebenwirkungen einer Botulinum therapie wie herabgesunkene Augenbrauen oder Doppeltsehen sind teilweise therapierbar und durch den Wirkungsverlust von Botulinum vollständig reversibel. Größere Studien ergaben, dass 99\% der Behandlungen mit Botulinum nebenwirkungsfrei verlaufen (Carruthers u. Carruthers 2009; Naumann u. Jancovic 2004).

Bei den Fillerbehandlungen mit Hyaluronsäure zeigen sich deutlich geringere Häufigkeiten von schweren Nebenwirkungen als bei Verwendung von permanenten oder semipermanenten Fillermaterialien. So treten die gefürchteten Femdkörperreaktionen bei Anwendung von Hyaluronsäuren deutlich seltener auf, wohingegen bei permanenten Füllmaterialien diese Reaktionen auch noch Jahre nach Applikation auftreten können und schwierig oder unzureichend therapierbar sind (Zielke et al. 2008; Bachmann et al. 2009). Reaktionen auf Hyaluronsäure verschwinden in den meisten Fällen durch Abbau des Produktes oder können mit Hylase ${ }^{\circledR}$ (Hyaluronidase) aufge- 
löst werden. Aus diesen Gründen sprechen sich sämtliche deutschen ästhetisch-chirurgischen Fachgesellschaften gegen die Verwendung von nicht abbaubaren Fillern aus.

Kontraindikationen für die Behandlung mit Botulinum und Fillern bestehen wenige. So sollten Schwangere und Stillende nicht behandelt werden, auch bei Autoimmunerkrankungen sollte nur nach vorheriger kritischer Abwägung behandelt werden. Unter 18 Jahren sollten ebenso keine Behandlungen mit Botulinum und Fillern durchgeführt werden.

Die wachsende Nachfrage nach den minimalinvasiven Methoden führt allerdings dazu, dass auch zunehmend nicht-ärztliche Berufsgruppen, wie Kosmetikerinnen oder Heilpraktiker diese Behandlungen durchführen. Weiterhin werden mit Botox-Flatrates, auch von ärztlichen Praxen, geworben und Dumpingpreise für Botulinumbehandlungen im Internet angeboten. Diese Entwicklung ist sicher kritisch zu betrachten und weiter zu beobachten.

Verschiedene Zentren, so das Zentralregister für Fillernebenwirkungen oder Fillerwelt, sammeln Daten zu Nebenwirkungen von allen dermalen Fillern, um spezielle Materialien zu identifizieren, die zu häufigen Komplikationen geführt haben und auch um entsprechende Therapieregimes zu erarbeiten.

\subsection{Fazit für den klinischen Umgang}

Insbesondere aufgrund der geringen Nebenwirkungen und der raschen und unkomplizierten Anwendung der minimalinvasiven Verfahren mit Botulinum und Fillern sowie der zunehmenden Qualität der Produkte nehmen die Behandlungszahlen $z u$.

Behandlungen mit Botulinum oder Fillern sind heute für viele Frauen und zunehmend auch für Männer eine nicht mehr wegzudenkende und normale Methode zur Verbesserung ihres Aussehens, vergleichbar einem regelmäßigen Kosmetik- oder Zahnarztbesuch.

Bei den elektiven ästhetischen Behandlungen mit Hyaluronsäuren und Fillern ist der Arzt in der Situation eines Dienstleisters. Der Wunsch des Patienten nach der Behandlung von Falten ist hier das Therapieziel.

Dabei muss der behandelnde Arzt genau eruieren, ob falsche Erwartungen oder etwa überzogene Vorstellungen bei dem Patienten vorliegen. Das Ergebnis kann, selbst vom erfahrenen Arzt, nicht immer hundertprozentig vorhergesagt werden.

Letztendlich gibt der Patient vor, ob er seine Mimik vollständig ausgeschaltet oder diese nur minimiert haben möchte. Auch mit wie viel Volumen z.B. die Lippe aufgefüllt werden soll, entscheidet der Patient. In der Verantwortung und in der ästhetischen Vorstellung des Arztes liegt es letztendlich, diesen 
Wünschen und Vorstellungen zu entsprechen, diese zu modifizieren oder eventuell auch abzulehnen.

Untersuchungen zu plastischen Operationen sehen den Anteil von psychisch auffälligen Personen mit BDD (body dysmorphic disorder), die ästhetische Operationen wünschen, bei bis zu 15\% (Harth u. Hermes 2007). In der dermatologischen Praxis soll der Anteil der Patienten mit psychischen Störungen noch höher sein. In einer Untersuchung von Atkan et al. lag dieser Anteil bei bis zu 33,4\% (Atkan et al. 1998) und bei bis zu 60\% in einer Untersuchung von Hughes et al. (1983). In der Normalbevölkerung sind es verschiedenen Studien zufolge $1-4 \%$.

Wie hoch die Zahlen an psychisch auffälligen Personen sind, die regelmäßig minimalinvasive Verfahren in Anspruch nehmen und ob dies dem Anteil der bei kosmetischen Operationen entspricht, dazu liegen bislang keine Untersuchungen oder Studien vor.

Bei den Schönheitsoperationen sind ein Drittel der Patienten im Alter zwischen 18-30 Jahren. Trifft dies auch auf die Behandlung mit Botulinum oder Fillern zu?

Stellt die Behandlungen mit Botulinum und dermalen Fillern eine sogenannte Einstiegsdroge oder einen leichteren Zugang für weitere ästhetische Maßnahmen zur Selbstverbesserung wie ästhetisch-plastische Schönheitsoperationen dar? Im Botox ${ }^{\circledR}$ Cosmetics Survey 2005 hatten 40\% der mit Botulinum behandelten Patienten schon eine kosmetische Operation durchführen lassen.

Ändert sich die Zufriedenheit des Patienten mit dem Ergebnis nach dem Wirkungsverlust von Botulinum oder dem Abbau der Hyaluronsäure? Will der zufriedene Patient immer mehr oder immer öfter eine Behandlung haben?

Soziologische oder psychologische Untersuchungen an dem großen Patientengut der Anwender von Botulinum und Fillern fehlen. Zurzeit wird in unserer und weiteren Arztpraxen in Zusammenarbeit mit der Universität Leipzig eine erste Untersuchung durchgeführt, mithilfe derer diese angesprochenen psychosozialen Fragestellungen insbesondere auch nach der Motivation für einen minimalinvasiven Eingriff geklärt werden sollen. Da noch keine auswertbaren Ergebnisse der Untersuchung vorliegen, ist noch keine ganz genaue Aussage möglich.

Bei Behandlung durch einen erfahrenen, gut aus- und weitergebildeten Arzt und bei Verwendung von in Studien geprüften Produkten ist die minimalinvasive Behandlung mit Botulinum und HS-Fillern ein sicheres und optimales Verfahren zur Verbesserung oder Minimierung von Alterserscheinungen des Gesichtes. Nach Selbstverbesserung des äußeren Erscheinungsbildes wurde schon immer gestrebt. Gegenüber früheren, teils gefährlichen Methoden, wie Injektionen von Silikonöl und Paraffin, stehen mit den heutigen Produkten sehr sichere Medikamente bzw. Medizinprodukte mit geringen Neben- 
wirkungen zur Verfügung. So wird man es den meisten Menschen nicht sofort ansehen, ob sie mit Botulinum oder/und Fillern behandelt sind. Sie wirken vielleicht nur für ihr Alter, sofern einem dies bekannt ist, jünger und vital aussehend. Auffallen wird einem nur die übertriebene und unnatürliche Anwendung.

\section{Literatur}

Aesthetic Surgery Education and Research Foundation (ASERF) (2005) BOTOX ${ }^{\circledR}$ Cosmetic Use Survey (unveröffentlichte Studie)

American Society for Aesthetic Plastic Surgery, ASAPS (2011) 15th annual cosmetic surgery national data bank statistics. Online unter: http://www.surgery.org/sites/default/files/ASAPS-2011-Stats.pdf (abgerufen am 02.10.2013)

American Society for Aesthetic Plastic Surgery, ASAPS (2010) cosmetic surgery national data bank statistics. Online unter: http://www.surgery.org/sites/default/files/Stats2010_1.pdf (abgerufen am 02.10.2013)

American Society for Aesthetic Plastic Surgery, ASAPS (2009) cosmetic surgery national data bank statistics. Online unter: http://www.surgery.org/sites/default/files/200gstats.pdf (abgerufen am 02.10.2013)

American Society of Plastic Surgeons - ASPS (2011) 2011 Plastic Surgery Procedural Statistics. Online unter: http://www.plasticsurgery.org/News-and-Resources/2011-Statistics-html (abgerufen am 02.10.2013)

Atkan S, Ozmen E, Sanili B (1998) Psychatric disorders in patient attending a dermatology outpatient clinic. Dermatology 197, 230-234

Bachmann F, Erdmann R, Hartmann V, Wiest L, Rzany B (2009) The spectrum of adverse reactions after treatment with injectable fillers in the glabellar region: results from the Injectable Filler Safety Study. Dermatol Surg 35(2), 1629-1634

Carruthers |, Carruthers A (1990) The treatment of glabellar furrows with botulinum A exotoxin. | Dermatol Surg Oncol 16, 83

Carruthers |, Carruthers A (2009) Botulinum toxin in facial rejunivation: an update. Dermatol Clin 27, 417-425

Gesellschaft für Ästhetische Chirurgie Deutschland e.V. - GÄCD (2011) Neue Statistik der Schönheitsoperationen. Online unter: http://www.gacd.de/fileadmin/user_upload/pdf/presse2011/Presseinformation_ |ahresstatistik_Schoenheitsoperationen.pdf (abgerufen am 02.10.2013)

Harth W, Hermes B (2007) Psychosomatic disturbances and cosmetic surgery. JDDG 5, 736-744

Hughes I, Barraclough B, Hamblin L, White I (1983) Psychatric symptons in dermatologic patients. Br | Psychatry $143,51-54$

Lewis MB, Bowler PJ (2009) Botulinum toxin cosmetics therapy correlates with a more positive mood. I Cosmetic Derm 8, 24-26

Naumann M, Jancovic I (2004) Safety of botulinum toxin type A: a systematic review and metaanalysis. Curr Med Res Opin 20, 981-990

Sommer B, Bergfeld D, Sattler G (2010) Botulinumtoxin in der ästhetischen Medizin. Thieme Verlag Stuttgart

Sommer B, Zschocke I, Bergfeld D, Sattler G, Augustin M (2003) Satisfaction of Patients after treatment with botulinum toxin for dynamic facial lines. Dermatol Surg 29, 444-449

Wiest LG (2007) Historie und Anwendung der Filler zur Faltenbehandlung. Hautarzt 58, 224-231

Wollmer MA, de Boer C, Kalak N, Beck J, Götz T, Schmidt T, Hodzic M, Bayer U, Kollmann T, Kollewe K, Sönmez D, Duntsch K, Haug MD, Schedlowski M, Hatzinger M, Dressler D, Brand S, Holsboer-Trachsler E, Kruger TH (2012) Facing depression with botulinum toxin: A randomized controlled trial. J Psychatric Res 46(5), 74-81

Zielke H, Wölber L, Wiest L, Rzany B (2008) Risk profiles of different injectable fillers: results from the injectable Filler Safety Study (IFS Study). Dermatol surg 34(3), 326-35 